\title{
Analysis of Half-Turn Effect in Power Transformers Using Nonlinear-Transient FE Formulation
}

\author{
G. B. Kumbhar, S. V. Kulkarni, Member, IEEE, and V. S. Joshi
}

\begin{abstract}
The half-turn effect is present in transformers if the winding leads are taken out from the different sides of the core. The result is an additional half-turn in one of the core windows in a single-phase transformer, which can create overfluxing of the core leading to excessive losses and temperature rises. This paper presents a methodology to analyze the half-turn effect in power transformers. A nonlinear field-circuit coupled model is used to simulate the half-turn effect; the magnetic field is modeled using the finite-element method. The results show that there is a considerable increase in the flux density values in the core during load loss test, and the increased core losses can be comparable to the values under the rated voltage condition. The results obtained by the proposed methodology are validated with the experimental test results on a single-phase 40-MVA autotransformer. The half-turn effect can be eliminated by taking both the leads from the same side of the core. However, in order to reduce insulation clearances, a few compensating turns are wound on the end limbs to eliminate the half-turn effect. The paper also reports the experimental and the simulation results with the compensating turns. Analysis of the high induced voltages in the unexcited windings of the other phases during the load-loss test on the middle phase of three-phase three-limb transformers is also reported. In the three-phase fivelimb transformers, the effects of half-turns in the three phases are almost cancelled under the balanced load conditions; this paper highlights the possibility of the core saturation due to the half-turn effect under appreciable unbalanced load conditions.
\end{abstract}

Index Terms-Field-circuit coupling, finite-element method (FEM), half-turn effect, nonlinear transient.

\section{INTRODUCTION}

$\mathbf{T}$ HE transformer is a very critical and costly piece of equipment in power systems. Its efficiency can be improved by accurate evaluation and reduction of losses. In some transformers, the leads of the high-voltage (HV) or the low-voltage (LV) winding may not be taken out from the same side of the core as shown in Fig. 1. This arrangement simplifies the connections, and results in lower insulation clearances. However, it leads to the presence of the half-turn in the winding. This phenomenon is particularly observed in single-phase autotransformers having three-limbed core construction, in which the HV and the LV bushings are fitted on the tank cover on the opposite

Manuscript received July 12, 2005; revised November 15, 2005. This work was supported by the Department of Science and Technology, Government of India, under Project SR/FTP/ET-165/2001. Paper no. TPWRD-00406-2005.

G. B. Kumbhar and S. V. Kulkarni are with the Indian Institute of Technology-Bombay, Mumbai 400076, India (e-mail: ganeshk@ee.iitb.ac.in; svk@ee.iitb.ac.in).

V. S. Joshi is with Transformer Division, Crompton Greaves Ltd., Mumbai 400042, India (e-mail: vikrant.joshi@cgl.co.in).

Digital Object Identifier 10.1109/TPWRD.2006.876663

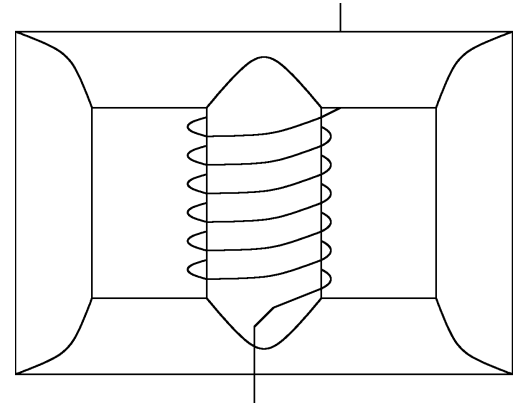

Fig. 1. Half-turn in single-phase three-limb transformer.

sides of the core. In this case, either the LV or the HV would contain a half-turn as the lead crosses the core to the opposite side for the termination. In double-wound transformers, the half-turn is present if the line and the neutral terminals are not on the same side of the core for any of the two windings. Even though this arrangement helps designers simplify the insulation design, it results in overfluxing and increased core losses, which can lead to excessive temperature rise in the transformer core [1].

In the case of the three-phase three-limb transformers, during the single-phase load-loss test on the middle phase, high voltages are induced in the windings of the other two phases due to the half-turn in one of the windings on the middle phase. The flux produced by the half-turn passes through the yokes and the extreme limbs and it is so large that these limbs can become saturated [2].

There is no published literature which has reported any in-depth and accurate analysis of the half-turn effect. Hence, the half-turn effect may come as a big surprise to those who manufacture for the first time a transformer with the presence of the half-turn in one of the windings. This paper gives a comprehensive analysis of the half-turn effect in commonly used core constructions, including single-phase three-limb, three-phase three-limb, and three-phase five-limb. From the perspective of power utilities, the results presented for the three-phase five-limb transformers are important. Operation with an appreciable unbalance could lead to serious overfluxing and temperature rise problems.

These days, numerical modeling techniques, which are well established, enable representation of all important phenomena occurring inside the transformers [3]. Field-circuit coupled formulation is commonly used in conjunction with a nonlineartransient electromagnetic-field model of devices [4], [5]. In this work, nonlinear-transient field-circuit coupled 2-D simulations are carried out to investigate the half-turn effect in the transformers. Nonlinearity is taken into account by using actual B-H 


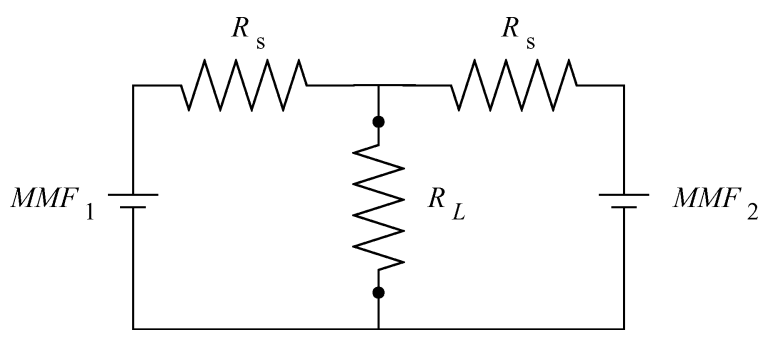

Fig. 2. Magnetic equivalent circuit.

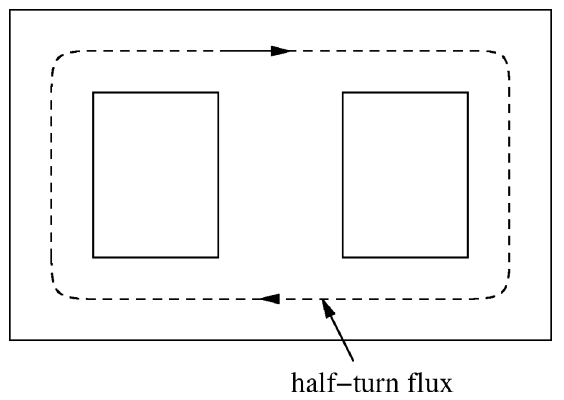

Fig. 3 Path of half-turn flux.

curve of the core material. This paper describes in detail the formulation, modeling, and analysis of the half-turn effect in the single phase as well as the three-phase transformers. The obtained FEM simulation results closely agreed with the experimental data.

\section{HALF-TURN EFFECT}

As shown in Fig. 1 for a single-phase transformer, when the leads of a winding are taken on the opposite sides of the core, the last turn is passed through a window with no return back. Hence, the numbers of turns passing through the two windows on the either sides of the middle limb are not equal. Due to the difference in the numbers of turns, the ampere-turns in both windows are different and do not compensate each other. Let $n_{1}$ and $n_{2}$ be the number of turns on the primary and the secondary windings of the single-phase three-limb transformer. Then, $\operatorname{MMF}_{1}\left(=\left(n_{1}-\right.\right.$ 1) $\left.I_{1}-n_{2} I_{2}\right)$ and $\mathrm{MMF}_{2}\left(=n_{1} I_{1}-n_{2} I_{2}\right)$ are the ampereturns related to the two windows under the load-loss test condition. Here, the magnetizing ampere-turns have been neglected. Fig. 2 shows the simplified magnetic equivalent circuit of the considered transformer. The unbalanced magnetomotive force (MMF), which has resulted from the difference between $\mathrm{MMF}_{1}$ and $\mathrm{MMF}_{2}$, generates a flux which flows through the yokes and the end limbs as shown in Fig. 3. Since the voltage source is connected to the primary winding with the secondary winding short-circuited, the middle limb does not allow any flux component to flow through it other than the no-load flux. It is apparent that the MMF acting on the flux path shown is $I_{1}$. This unbalanced MMF can be substantial and comparable to the magnetizing ampere-turns which set up the rated flux density in the yokes and the end limbs during the no-load test. At site, the half-turn flux, superimposed over the no-load flux, can lead to overfluxing of the core.

\section{COUPled FiEld-CirCUIT Formulation}

\section{A. Electromagnetic Model}

For 2-D magnetic analysis, consider the classical Poisson's equation

$$
\frac{\partial}{\partial x}\left(\frac{1}{\mu} \frac{\partial A_{z}}{\partial x}\right)+\frac{\partial}{\partial y}\left(\frac{1}{\mu} \frac{\partial A_{z}}{\partial y}\right)=-J_{z}
$$

where $A_{z}$ and $J_{z}$ denote the $z$ components of the magnetic vector potential and the free or the source current density, respectively, and $\mu$ is the material permeability.

It is assumed that the windings are made up of thin stranded conductors. These thin stranded conductors in the windings can be modeled with uniform current densities (i.e., the induction term in the magnetic-field equations representing eddy currents is neglected). Assuming that each turn carries $I$ amperes of current, the magnitude of current density of a winding is

$$
J_{z}=d \frac{n I}{S_{c}}
$$

where $n$ and $S_{c}$ are the number of turns and the cross-sectional area of the winding, respectively, and $d$ is the polarity $(+1$ or $-1)$ to represent the forward or the return path. After space discretization of (1) using Galerkin method [6], we have

$$
[K]\{A\}+[D]\{I\}=0
$$

where

$$
\begin{aligned}
& {[K]=\sum_{\Omega} \frac{1}{\mu} \iint_{\Delta_{e}}\left(\frac{\partial N_{e}^{T}}{\partial x} \frac{\partial N_{e}}{\partial x}+\frac{\partial N_{e}^{T}}{\partial y} \frac{\partial N_{e}}{\partial y}\right) d x d y} \\
& {[D]=\sum_{\Omega} \frac{n}{S_{c}} \iint_{\Delta_{e}} N_{e}^{T} d x d y}
\end{aligned}
$$

where $N_{e}$ and $\Delta_{e}$ are the element shape function and the element area, respectively, and $\Omega$ indicates the whole domain. $N_{e}^{T}$ represents the transpose of the matrix $N_{e}$.

\section{B. External Circuit Equations}

The matrix form of external circuit equations can be written as

$$
[U]=\left\{\frac{d \Phi}{d t}\right\}+[R]\{I\}+[L]\left\{\frac{d I}{d t}\right\} .
$$

If flux linkages are expressed in terms of the magnetic vector potential, the matrix form of the external circuit equations can be written as

$$
[U]=[G]\left\{\frac{d A}{d t}\right\}+[R]\{I\}+[L]\left\{\frac{d I}{d t}\right\}
$$

where $U$ is the vector of input voltages, $[L]$ and $[R]$ are the matrices of leakage inductances and winding resistances, respectively, and $G$ is a matrix similar to $D$, which depends on the geometrical features of the windings. 


\section{Nonlinear-Transient Field-Circuit Coupled Model}

From (3) and (5), the field-circuit coupled global system of equations can be written as

$$
\left[\begin{array}{cc}
0 & 0 \\
G & L
\end{array}\right]\left\{\begin{array}{c}
\dot{A} \\
\dot{I}
\end{array}\right\}+\left[\begin{array}{cc}
K & D \\
0 & R
\end{array}\right]\left\{\begin{array}{c}
A \\
I
\end{array}\right\}=\left\{\begin{array}{c}
0 \\
U
\end{array}\right\} .
$$

In this system of equations, the unknowns are the nodal values of the magnetic vector potentials $(A)$ and the currents in the external circuits $(I)$. To solve this time-dependent system of equations, a numerical integration scheme such as Euler Backward or Crank-Nicholson algorithm can be used. The nonlinearities in the modeled system are taken into account by using the Newton-Raphson iterative procedure. The resulting system of equations is

$$
\begin{aligned}
& {\left[\begin{array}{cc}
\beta\left(K^{n}+K_{n l}^{n}\right) & \beta D \\
\frac{G}{\Delta t} & \beta R+\frac{L}{\Delta t}
\end{array}\right]\left\{\begin{array}{c}
\Delta A \\
\Delta I
\end{array}\right\}_{t+\Delta t}^{n+1}} \\
& =\left\{\begin{array}{cc}
0 \\
\beta V_{t+\Delta t}+(1-\beta) V_{t}
\end{array}\right\} \\
& +\left[\begin{array}{cc}
-(1-\beta) K & -(1-\beta) D \\
\frac{G}{\Delta t} & -(1-\beta) R+\frac{L}{\Delta t}
\end{array}\right]\left\{\begin{array}{c}
A \\
I
\end{array}\right\}_{t} \\
& \quad-\left[\begin{array}{cc}
\beta\left(K^{n}\right) & \beta D \\
\frac{G}{\Delta t} & \beta R+\frac{L}{\Delta t}
\end{array}\right]\left\{\begin{array}{c}
A \\
I
\end{array}\right\}_{t+\Delta t}^{n}
\end{aligned}
$$

where the term $K_{n l}^{n}$ accounts for the nonlinearity of the core material. The value of $\beta$ determines the nature of the time-stepping scheme [6].

\section{RESULTS AND DisCUSSIONS}

\section{A. Single-Phase Three-Limb Transformer}

A 40-MVA, $(220 / \sqrt{3}) /(132 / \sqrt{3}) / 33 \mathrm{kV}$, single-phase three-limb autotransformer is analyzed to investigate the half-turn effect and the effectiveness of compensating turns. The required geometrical dimensions of the analyzed transformer are shown in Fig. 4(a). The equivalent cross-sectional area of the middle limb of the core is $2679 \mathrm{~cm}^{2}$. The design values of volt/turn, flux density, and core losses are $99.7 \mathrm{~V}$, $1.675 \mathrm{~T}$, and $19.59 \mathrm{~kW}$, respectively. The HV series winding of the autotransformer has $3941 / 2$ turns (thus, having the half turn in it) with the rated current of $314.9 \mathrm{~A}$.

The FEM simulations are performed using the 2-D rectangular Cartesian coordinate system. In this particular case, the axisymmetric system cannot be used since the simulation of the half-turn effect requires modeling of both the core windows with different ampere-turns. Also, the 2-D Cartesian system is more appropriate because the flux due to the half-turn effect flows through the yokes and the end limbs. In order to simulate the half-turn effect, one extra turn is introduced in the HV winding in one of the windows in the finite-element analysis. The core is assumed to have a rectangular cross section having the same cross-sectional area as that of the actual core. Therefore, the core stack height considered for the simulation is $43.21 \mathrm{~cm}$. The windings are connected to represent conditions during the load loss measurement test and the required voltage is applied across the LV (132-kV) winding [Fig. 4(b)]. The value of the voltage applied is such that the required currents flow through the HV

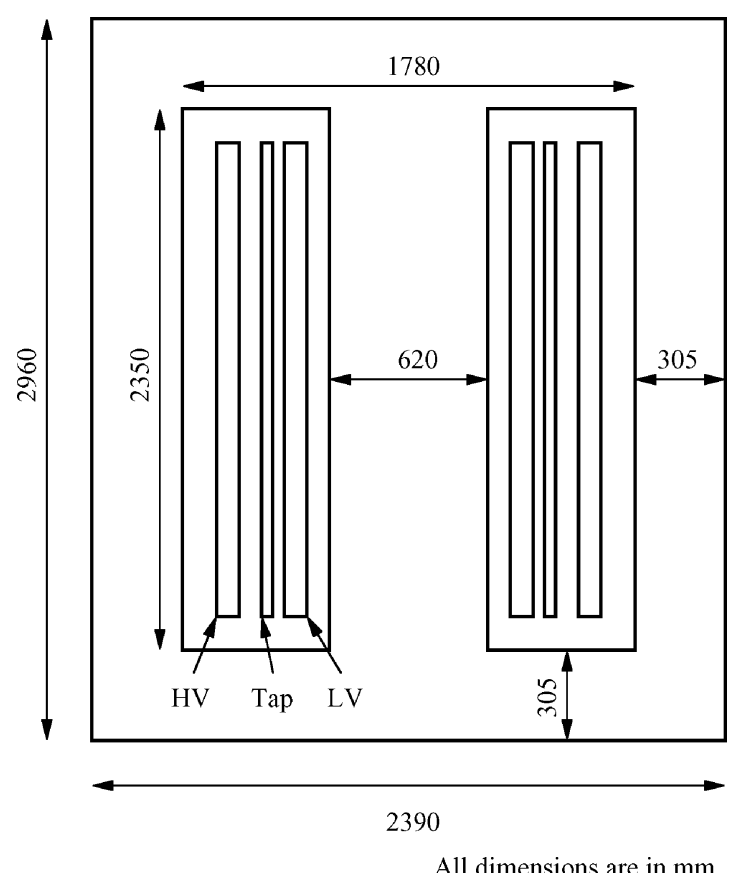

(a)

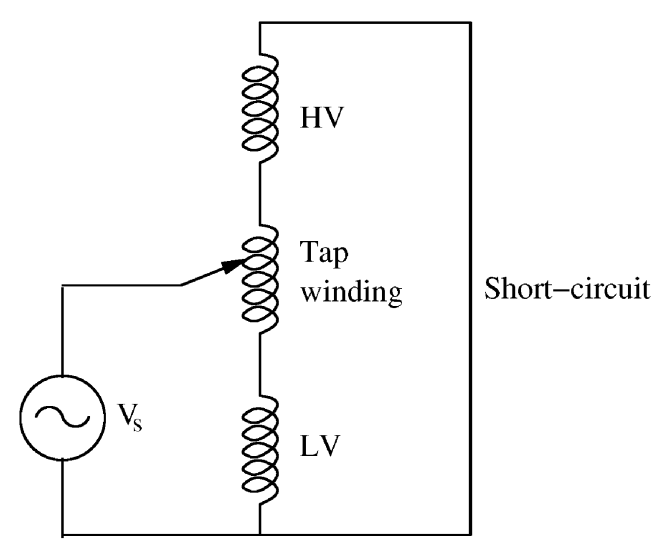

(b)

Fig. 4. (a) Analyzed geometry and (b) connection diagram at load loss test.

and the LV windings with the HV winding short-circuited. Nonlinear-transient field-circuit coupled finite-element analysis is carried out using the code developed in MATLAB based on the methodology given in Section III. The developed code has been verified using commercial FEM software. The nonlinearity of the core material is taken into account by using the $\mathrm{B}-\mathrm{H}$ curve of the $\mathrm{M} 3 \mathrm{H}$ material corresponding to the built core condition. The transformer is simulated with and without the half-turn by adjusting the number of turns in the two windows.

First, the transformer is simulated under a load loss test condition with the rated currents flowing in the HV $(220-\mathrm{kV})$ and the LV (132-kV) windings. Due to the half-turn effect, the flux density established in the outer path (formed by the yokes and the end limbs) is $1.61 \mathrm{~T}$. The corresponding additional loss in the yokes and the end limbs has been calculated as $11.7 \mathrm{~kW}$ which is comparable to the rated no-load loss value of $19.59 \mathrm{~kW}$ for the whole core. The flux density distribution computed from the field-circuit finite-element model is used to determine the core 


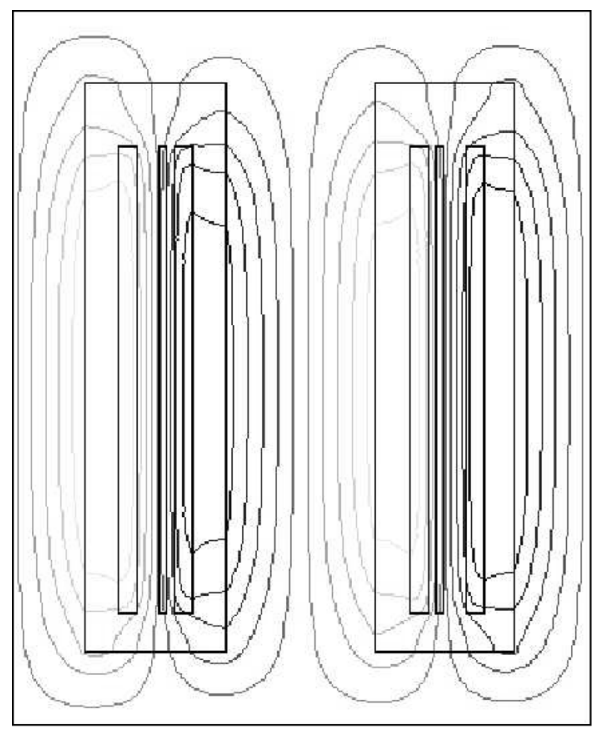

$\max : 0.00382 \mathrm{Weber} / \mathrm{m}$

(a)

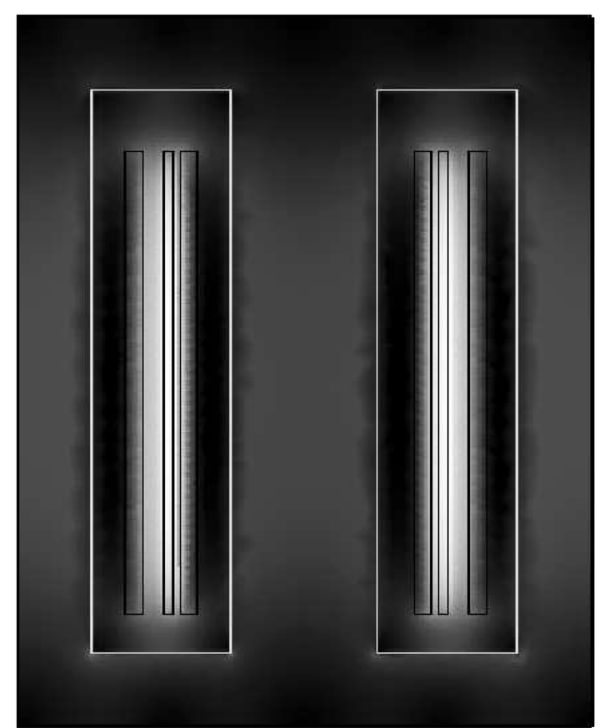

$\max : 0.037$ Tesla

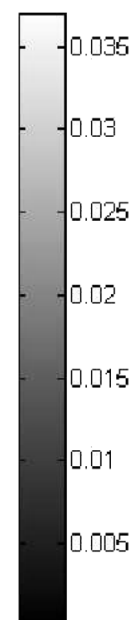

$\min : 3.32 e-005$ Tesla

(b)

Fig. 5. (a) Flux distribution and (b) flux density plots without half-turn.

TABLE I

COMPARISON BETWEEN MEASURED AND CALCUlated VALUES AT 50\% OF RATED CURRENT

\begin{tabular}{|l|c|c|}
\hline \hline & Measured value & Calculated value \\
\hline \hline $\begin{array}{l}\text { Flux density in end } \\
\text { limbs in Tesla }\end{array}$ & 1.04 & 0.93 \\
\hline $\begin{array}{l}\text { Extra core loss by the } \\
\text { half-turn in kW }\end{array}$ & 4.2 & 3.9 \\
\hline \hline
\end{tabular}

loss in the yokes and the end limbs. The flux density versus watts per kilogram curve for $\mathrm{M} 3 \mathrm{H}$ (core material) is used to compute the loss distribution. Cubic spline technique is used to obtain smoothness in 2-D interpolation. The loss in an individual element is calculated from its weight and the value of flux density in it. The total loss is computed by integrating the losses of all the elements.

In order to validate the developed methodology of analysis, experimental verification has been done on the 40-MVA transformer. For the convenience of testing, the load loss test was done at $50 \%$ of the rated current. Also, to obtain the flux density values in the yokes and the end limbs, a few turns were wound on them. The measured value of the voltage across these turns was used to obtain the flux density value. The corresponding results of the finite-element method (FEM) simulation (at $50 \%$ of the rated current) are compared with the test results as shown in Table I. The increase in the core loss due to the HV half-turn has been determined experimentally by repeating the test with the HV lead taken through the window to the other side of the core so that the half-turn is eliminated. Fig. 5(a) and (b) shows the leakage flux lines (Weber per meter) and flux density (Tesla) distribution, respectively, in the core without the half-turn at steady state (i.e., few cycles after decay of initial transients in the simulation). The average value of the flux density in the end limbs of the core is about $0.011 \mathrm{~T}$. With the presence of the half-turn, the additional 1 ampere-turn acts on the core of the transformer. The flux produced by this ampere-turn flows through the yokes and the two end limbs. The middle limb does not allow this flux to flow through it due to the presence of the voltage source connected to the primary winding with the secondary winding short circuited. The flux distribution due to the half-turn effect is shown in Fig. 6(a). Some flux lines are seen in the top and the bottom parts of the middle limb since the short-circuited winding does not cover the whole window height. There is a considerable amount of increase in the values of flux densities as shown in Fig. 6(b). The average value of flux density in the yokes and the end limbs is $0.93 \mathrm{~T}$. The measured value is $1.04 \mathrm{~T}$.

The circulating flux due to the half-turn effect could lead to undesirable core saturation conditions at site under the rated voltage and the rated current condition. In this case, the half-turn flux superimposes on the main (mutual) flux saturating the core. When the above autotransformer is simulated under the rated voltage and the rated current condition, the flux density in the yokes and the end limbs has been obtained as $1.96 \mathrm{~T}$ which is close to the saturation value of $2.0 \mathrm{~T}$.

The half-turn effect is commonly eliminated in single-phase transformers by winding a few compensating turns on the end limbs as shown in Fig. 7 [1]. The half-turn effect is nullified; there is no additional (core) loss during the load loss test, except for a small $I^{2} R$ loss in the compensating turns. The flux components in the two end limbs, due to the half-turn effect, are in antiphase. This leads to induction of the electromotive-force (emf) components which are antiphase in the two compensating windings, circulating a current in the closed loop. If $n$ is the number of turns of each compensating winding, then the value of the current flowing through the loop is $I_{1} / 2 n$. A simulation has been carried out with the presence of the compensating turns on the end limbs. The measured and the computed values of the current are given in Table II.

\section{B. Three-Phase Three-Limb Transformers}

The half-turn effect, if present, becomes clearly evident in the three-phase three-limb transformers during the ratio test which is done phase-by-phase. For an arrangement of the three phases 


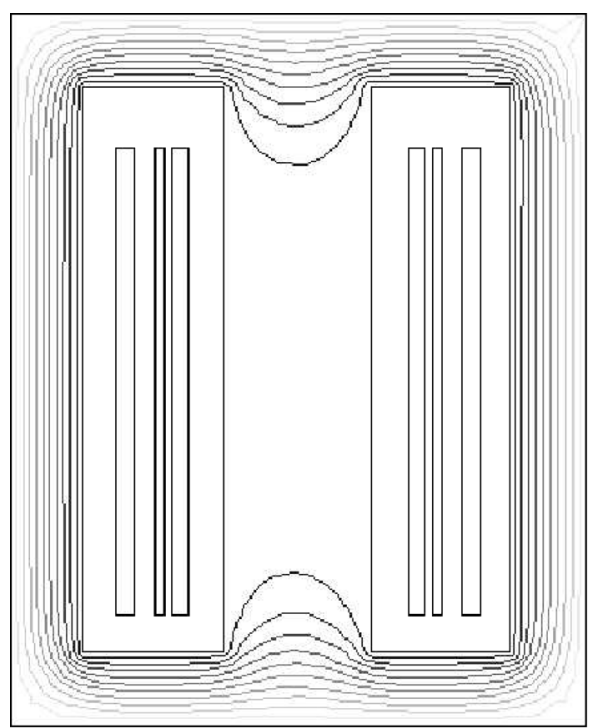

$\max : 7.92 \mathrm{e}-010 \mathrm{Weber} / \mathrm{m}$

(a)
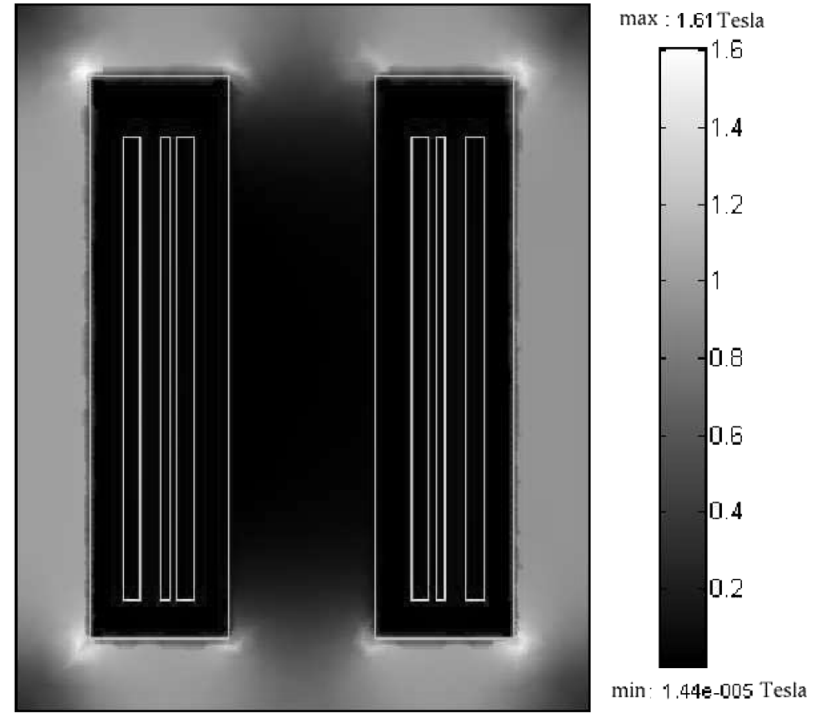

(b)

Fig. 6. (a) Flux distribution and (b) flux density plots with half-turn.

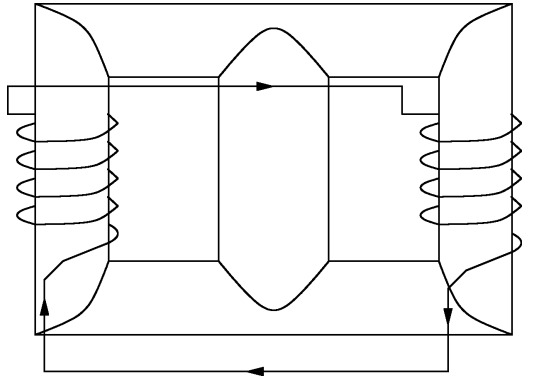

Fig. 7. Compensating turns.

TABLE II

Comparison Between Measured and Calculated Values of CURRENT IN COMPENSATING TURNS

\begin{tabular}{|l|c|c|}
\hline \hline & Measured value & Calculated value \\
\hline \hline $\begin{array}{l}\text { Current in compensating } \\
\text { turns in Amps }\end{array}$ & 79 & 78.5 \\
\hline \hline
\end{tabular}

A, B, C from the left to the right with turns (say, $4001 / 2$ ) of the windings wound clockwise, the turns ratio measured for the $\mathrm{A}, \mathrm{B}$, and C phases corresponds to 400, $4001 / 2$, and 401 turns, respectively [1].

However, the three-phase three-limb transformers are not affected by the half-turn effect under a three-phase excitation during the load loss test. This is because of the absence of the outer low reluctance path (which exists in the single-phase three-limb case). The presence of the voltage sources on all three limbs does not allow the half-turn flux to flow through them. Also, the effects of the three half-turn fluxes of the three phases are cancelled in the yokes under balanced three-phase conditions. In the case of unbalanced loading conditions, the resultant non-zero ampere-turns due to the half-turn effect of the three phases have to overcome an extremely high reluctance
TABLE III

RESUlTS FOR THREE-PHASE THREE-LIMB TRANSFORMER

\begin{tabular}{|c|c|c|c|c|c|}
\hline \hline & \multirow{2}{*}{$\begin{array}{c}\text { Flux density } \\
\text { in yokes } \\
\text { and extreme }\end{array}$} & \multicolumn{4}{|c|}{$\begin{array}{c}\text { Magnitude of voltage induced } \\
\text { in extreme phases in } \mathrm{kV}\end{array}$} \\
\cline { 3 - 6 } & & \multicolumn{2}{|c|}{ A phase } & \multicolumn{2}{|c|}{ C phase } \\
\cline { 3 - 6 } & limbs in Tesla & LV & HV & LV & HV \\
\hline \hline Without half-turn & 0.004 & 0.07 & 0.31 & 0.07 & 0.31 \\
\hline With half-turn & 1.16 & 22.50 & 90.09 & 22.74 & 90.33 \\
\hline \hline
\end{tabular}

of the nonmagnetic parts outside the core. Hence, the flux density induced in this path (formed by the yoke and the outside nonmagnetic path) is negligible (almost zero).

However, the half-turn effect has one particular significance for the three-phase three-limb transformers. During the phase-by-phase load loss measurement test, the losses measured in the middle phase are much higher than those of the extreme phases due to additional losses occurring in the available outer path formed by the yokes and the extreme limbs. These higher losses should not be interpreted as indicative of some problem in the middle phase. For the extreme phases, the outer path involves the other two limbs on one side and high reluctance nonmagnetic path on the other side. Hence, there is a negligible half-turn effect. The voltages induced in the open-circuited windings on the extreme limbs are quite high during the test on the middle phase. To analyze this phenomenon, a 31.5-MVA, 132/33-kV, YNd1 transformer is simulated using nonlinear-transient field-circuit coupled formulation. The induced voltages with and without the presence of the half-turn are shown in Table III.

\section{Three-Phase Five-Limb Transformers}

The half-turn effect in the three-phase five-limb transformers is found to be harmless since the three fluxes (corresponding to the three phases) tend to cancel each other being displaced in phase by $120^{\circ}$. This results in no additional core loss during the 
TABLE IV

RESUlTS FOR THREE-PHASE FIVE-LIMB TRANSFORMER

\begin{tabular}{|c|c|c|c|}
\hline \hline \multirow{2}{*}{} & \multicolumn{3}{|c|}{$\begin{array}{c}\text { Flux density in Tesla for } \\
\text { unbalanced currents in windings }\end{array}$} \\
\cline { 2 - 4 } & Balanced & $10 \%$ unbalance & $20 \%$ unbalance \\
\hline \hline Without half-turn & 0.02 & 0.035 & 0.045 \\
\hline With half-turn & 0.04 & 0.108 & 0.25 \\
\hline \hline
\end{tabular}

three-phase load loss test under balanced load conditions. Under appreciable unbalanced conditions, there would be a considerable amount of flux induced in the yokes and the end limbs, causing substantial additional core loss. The effect depends on the amount of unbalance between the three phases. The obtained simulation results on a 315-MVA three-phase five-limb transformer are shown in Table IV. This additional component of the flux density, when superimposed on the no-load flux density during normal service conditions, could lead to an overfluxing of the core. The three-phase five-limb transformer may be equipped with a delta-connected stabilizing winding. However, the presence or the absence of the stabilizing winding does not affect the phenomenon.

The half-turn effect in the three-phase five-limb transformers, which becomes significant under appreciable unbalanced conditions, cannot be eliminated by the arrangement of the compensating turns on the end limbs as shown in Fig. 7. This is because the main fluxes in the two end limbs are not in phase [1].

\section{CONCLUSION}

This paper has presented a detailed formulation, modeling and analysis of the half-turn effect in the single-phase and the three-phase transformers. A field-circuit coupled nonlinear finite-element model has been used to investigate the consequences of the half-turn effect. The model of a 40-MVA, $(220 / \sqrt{3}) /(132 / \sqrt{3}) / 33-\mathrm{kV}$ single-phase autotransformer was used to study the half-turn effect. The flux produced by the half-turn effect flows through the yokes and the end limbs. This flux can be so large that a high flux density (close to the rated value) can be established in the yokes and the end limbs. This could lead to overfluxing, excessive loss, and temperature rise in the core. The loss distribution in the core was computed using the flux density versus watts per kilogram curve of the material used to build the core. The simulation results obtained are in close agreement with the experimental test results. The half-turn effect, if present, is eliminated by using compensating turns wound on the two end limbs. The simulation and the experimental results with the compensating turns also closely agreed with each other.

In the three-phase three-limb transformers, during the singlephase load loss measurement test on the middle phase, the load loss value obtained is more than that of the other two phases.
Also, excessive voltages are induced in the windings on the extreme limbs.

In the case of three-phase five-limb transformers, the half-turn effects are cancelled due to the phasor addition of the three fluxes which are displaced by $120^{\circ}$ with each other in balanced conditions. Under appreciable unbalanced conditions, there would be a significant amount of flux induced in the yokes and the end limbs, causing substantially increased core loss and temperature rise.

\section{ACKNOWLEDGMENT}

The authors would like to thank the management of Crompton Greaves for permitting reference of the test results in this paper.

\section{REFERENCES}

[1] S. V. Kulkarni and S. A. Khaparde, Transformer Engineering: Design and Practice. New York: Marcel Dekker, May 2004.

[2] H. Mohseni and F. Hamdad, "Overvoltages caused by winding terminal arrangement of three phase transformer," in Proc. 9th Int. Symp. High Voltage Engineering, Aug./Sep. 1995, pp. 6757/1-6757/4.

[3] R. Tang, S. Wang, L. Yan, X. Wang, and C. Xiang, "Transient simulation of power transformers using 3D finite element model coupled to electric circuit equations," IEEE Trans. Magn., vol. 36, no. 3, pp. 1417-1420, May 2000.

[4] J.-S. Wang, "A nodal analysis approach for 2D and 3D magnetic-circuit coupled problems," IEEE Trans. Magn., vol. 32, no. 3, pp. 1074-1077, May 1996.

[5] I. A. Tsukerman, A. Konrad, and J. D. Lavers, "A method for circuit connections in time-dependent eddy current problems," IEEE Trans. Magn., vol. 28, no. 2, pp. 1299-1302, Mar. 1992.

[6] M. V. K. Chari and S. J. Salon, Numerical Methods in Electromagnetism. New York: Academic, 2000.

G. B. Kumbhar received the B.E. degree in electrical engineering from the Government College of Engineering, Maharashtra, India, in 1999 and the M.Tech degree from the Indian Institute of Technology-Madras, Chennai, India, in 2002.

Currently, he is a Research Scholar with the Indian Institute of Technology-Bombay, Mumbai, India, working in coupled field formulations in electrical machinery.

S. V. Kulkarni (M'99) is Associate Professor in the Department of Electrical Engineering, Indian Institute of Technology, Bombay, India. Previously, he was with Crompton Greaves Ltd., Mumbai, India, and specialized in the design and development of transformers up to the $400-\mathrm{kV}$ class. His research interests include transformer design and analysis, computational electromagnetics, and distributed generation. He has authored the book Transformer Engineering: Design and Practice (Marcel Dekker, 2004).

Dr. Kulkarni is the recipient of the Young Engineer Award (2000) from the Indian National Academy of Engineering.

V. S. Joshi received the B.E. degree from Bombay University, Bombay, India, in 1988 .

Currently, he is a Deputy General Manager of Design with the Transformer Division, Crompton Greaves Ltd., Mumbai, India. His research interests include the design and development of high-voltage and special transformers. 